\title{
Development of Non-Dissipative Numerical Schemes for Computational Aeroacoustics
}

\author{
Jeffrey P. Thomas* and Philip L. Roe ${ }^{\dagger}$ \\ Department of Aerospace Engineering \\ University of Michigan \\ Ann Arbor, MI 48109-2140
}

\begin{abstract}
The ability of popular computational fluid dynamic (CFD) methods to simulate the propagation of acoustic waves is assessed both theoretically and by numerical experiment. The schemes are used to model acoustic waves generated by a noise source situated at the aft end of a convergent-divergent nozzle. It is found that the acoustic signal that traverses up the nozzle has difficulty maintaining its correct amplitude as the frequency of the noise increases. When the sound is of sufficiently high frequency, the acoustic wave is dissipated so much that it does not reach the front end of the channel.

Based on this experience, we argue for schemes that are inherently free of dissipation. In one dimension, we study an "upwind" form of the leapfrog scheme, and show how it can be used to compute a linearized perturbation of the nonlinear solution obtained from any Euler code. This method is non-dissipative and can accurately model the correct amplitude of the acoustic wave no matter what the frequency of its source. The scheme is extended to multiple space dimensions by employing bicharacteristic equations defined for a certain 'staggered' storage of the unknowns. It remains free from dissipation, and the solution for the waves propagated into a half space by an oscillating piston is in excellent agreement with the analytical solution.
\end{abstract}

\section{Introduction}

CFD has become a powerful tool for simulating many types of flows, even those that are difficult or impossible to reproduce experimentally. As the power of current computer systems increase, the size of the of the problem one is able to model increases also. One type of fluid dynamics problem that is now becoming a possibility for CFD is that of aeroacoustics. Most practical problems in aeroacoustics are characterized by domains that span hundreds of wavelengths in three space dimensions, and

\footnotetext{
*Doctoral Pre-candidate, Aerospace Engineering

${ }^{\dagger}$ Professor, Aerospace Engineering
}

Copyright (C) 1993 by the American Institute of Aeronautics and Astronautics, Inc. All rights reserved. hence demand the large computational resources that are just becoming available (Reference [1]).

The requirement placed on the algorithm is to propagate weak waves over many wavelengths with minimal dispersion or dissipation. This contrasts with the requirement in much of 'mainstream' aerospace CFD to reach steady states by either damping out, or removing from the domain, the transient disturbances created during the start-up of the integration. Accurate handling of transients has of course received much attention from those concerned with the direct simulation of turbulence, where both spectral [2] and compact [3] differencing schemes are popular. At present, however, such schemes are not readily applicable to complex geometries.

In the aeroacoustic context, a very relevant parameter is the number $(N)$ of mesh points required per wavelength to hold dissipation and dispersion within acceptable bounds. Consider a computation in which waves of a given wavelength have to be propagated over a range of $R$ wavelengths. As targets for accuracy, we will assume that the position of a wave will be required to within one percent, and its amplitude to within ten percent. For a second-order method, the phase error is of the form

$$
\frac{\text { numerical wavespeed }}{\text { exact wavespeed }} \simeq 1-\frac{f(\nu)}{N^{2}}
$$

where $\nu$ is the CFL number, and $f(\nu)$ is a constant of order unity. For most methods, achieving one percent accuracy requires $N \simeq 20-25$, and the computing resources needed grow like $N^{4}$.

The amplitude poses an even more severe requirement. The attenuation per timestep will be

$$
g \simeq 1-\frac{g(\nu)}{N^{2}}
$$

where again $g(\nu)$ is of order unity. The number of timesteps required is $R N / \nu$, and so the total attenuation will be

$$
g^{R N / \nu} \simeq 1-\frac{g(\nu)}{\nu} \frac{R}{N}
$$

This shows that $N$, the points per wavelength required, grows linearly with $R$. The computing resources needed, namely $R \times N$ grid points in each Tirection, and a similar number of timesteps, grows like $R^{4} \times N^{4}$ ! 


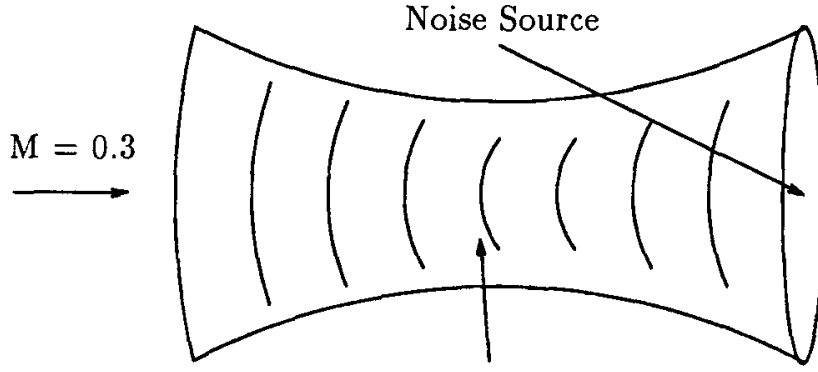

Acoustic Waves

Figure 1: Acoustic Waves in a Converging-Diverging Nozzle

Our aim in this paper is to develop methods for which there is no dissipation. In classical CFD this is not a desirable objective, because such schemes cannot handle shockwaves, but in the acoustic context shockwaves frequently do not arise. In itself, eliminating dissipation is easy; the classical leapfrog method does the job. However, we also aim to keep small the number $N$ dictated by acceptable phase error.

We begin by presenting a simple test problem consisting of a one-dimensional nozzle with a weak sound source at the exit. After sufficient time, the sound propagating forward should have equal amplitude at points that are symmetric with respect to the throat. However, at the throat, where the sound has to penetrate a nearly sonic flow, the spatial wavelength is much reduced, and the grid points there are not adequate to resolve it. In fact, we find that most 'aerospace' methods will attenuate the wave so much that frequencies above some critical value cannot reach forward of the throat.

We then present a new type of algorithm, that offers the low phase errors typical of high-order upwind schemes, without any dissipation. We analyze the scheme as applied to linear advection, and then show how it can be applied to computing perturbations of the nozzle flow, where the steady nonlinear solution has been obtained from any available Euler code.

Lastly we describe a multidimensional implementation of these ideas, applied to a 'pure' acoustics problem, i.e. one in which linear waves propagate through an uniform atmosphere.

\section{A Model Problem}

To demonstrate the difficulty of using dissipative numerical schemes for aeroacoustics, consider the onedimensional unsteady flow through a converging diverging nozzle. The nozzle has a parabolic shape with the area at the center of the channel equal to one half of that at each end (Figure 1). The inflow Mach number is 0.3 , so that the flow is subcritical everywhere, but reaches a Mach number of 0.86 at the throat.

A pressure source oscillating at a specified fre- quency and having an amplitude of $10^{-5}$ that of the inlet pressure is set at the aft end of the nozzle. The pressure source thus produces an acoustic wave that travels forward. Since the nozzle is symmetric about its midpoint, the amplitude of the wave that reaches the front end of the channel should eventually equal that of the pressure source that created it even though the amplitude of the wave may vary elsewhere in the nozzle.

\section{Nonlinear Solutions}

The conservation form of the one-dimensional variable area Euler equations can be expressed as

$$
\frac{\partial \mathrm{U}}{\partial t}+\frac{\partial \mathbf{F}}{\partial x}=\mathrm{T} \frac{d S}{d x}
$$

, where the conservation variables $U$, flux vector $\mathbf{F}$, and source vector $\mathbf{T}$ are given by

$$
\mathbf{U}=\left\{\begin{array}{c}
\rho S \\
\rho u S \\
E_{t} S
\end{array}\right\}, \mathbf{F}=\left\{\begin{array}{c}
\rho u S \\
\left(p+\rho u^{2}\right) S \\
\left(E_{t}+p\right) u S
\end{array}\right\}, \mathbf{T}=\left\{\begin{array}{l}
0 \\
p \\
0
\end{array}\right\} .
$$

Here, $\rho, u, p$, and $E_{t}$ represent dependent variables of density, velocity, pressure, and total energy respectively, and $S=S(x)$ is the cross-sectional area of the nozzle as a function of the spatial coordinate $x$. Assuming a calorically perfect gas, total energy is related to the other dependent variables via

$$
E_{t}=\frac{p}{\gamma-1}+\frac{\rho u^{2}}{2} .
$$

In order to demonstrate the problems of using regular aerospace schemes for aeroacoustics, two very different CFD methods commonly used for unsteady flow have been chosen. Both schemes are based upon the finitevolume method where an update for the conservative variables (see Equations 1a and 1b) at a mesh point $j$ is given by

$$
\mathrm{U}_{j}^{n+1}=\mathrm{U}_{j}^{n}-\frac{\Delta t}{\Delta x}\left(\mathbf{F}_{j+\frac{1}{2}}-\mathbf{F}_{j-\frac{1}{2}}\right)+\Delta t \mathbf{T}_{j}\left(\frac{d S}{d x}\right)_{j}
$$

\subsection{MacCormack's Method}

In MacCormack's method (Reference [4]) the interface flux at $j+\frac{1}{2}$ is determined using the following formulation

$$
\mathbf{F}_{j+\frac{1}{2}}=\frac{1}{2}\left(\mathbf{F}\left(\mathbf{U}_{j}^{n+\frac{1}{2}}\right)+\mathbf{F}\left(\mathbf{U}_{j+1}^{n}\right)\right)
$$

, with

$$
\begin{aligned}
\mathbf{U}_{j}^{n+\frac{1}{2}}=\mathbf{U}_{j}^{n} & -\frac{\Delta t}{\Delta x}\left(\mathbf{F}\left(\mathbf{U}_{j+1}^{n}\right)-\mathbf{F}\left(\mathbf{U}_{j}^{n}\right)\right) \\
& +\Delta t \mathbf{T}_{j}^{n}\left(\frac{d S}{d x}\right)_{j}
\end{aligned}
$$


The source term for Equation 2 is evaluated as

$$
\mathbf{T}_{j}=\mathbf{T}\left(\mathbf{U}_{j}^{n+\frac{1}{2}}\right)
$$

Since there are no shocks involved with the model problem that is under consideration, the artificial dissipation terms that are normally added to handle such situations have not been needed here. Boundary conditions are handled using the method of locally one-dimensional Riemann invariants (Reference [5]).

\subsection{An Upwind Method}

For Roe's approximate Riemann solver (Reference [6], an interface flux at $j+\frac{1}{2}$ is determined using

$\tilde{\mathbf{F}}_{j+\frac{1}{2}}=\frac{1}{2}\left(\tilde{\mathbf{F}}\left(\tilde{\mathbf{U}}_{L}\right)+\tilde{\mathbf{F}}\left(\tilde{\mathbf{U}}_{R}\right)\right)-\frac{1}{2}\left|\tilde{A}\left(\tilde{\mathbf{U}}_{L}, \tilde{\mathbf{U}}_{R}\right)\right| \Delta \tilde{\mathbf{U}}$

Here the conservative variables and flux terms ( $\tilde{U}$ and $\tilde{\mathbf{F}})$ are the same as in Equation $1 \mathrm{~b}$ with the exception that the area terms $S$ are not present. $\tilde{A}$ is the dissipation matrix as discussed in Reference [6], and $\Delta \tilde{U}$ is the difference between the right and left states $\left(\tilde{U}_{R}-\tilde{U}_{L}\right)$.

For the first order method, the left and right states are simply chosen as the states within the cells adjacent to the interface. Namely,

$$
\tilde{\mathbf{U}}_{L}=\tilde{\mathbf{U}}_{j}^{n} \quad \text { and } \quad \tilde{\mathbf{U}}_{R}=\tilde{\mathbf{U}}_{j+1}^{n} .
$$

For the time-accurate second order method, Hancock's reconstruction technique (Reference [7]) is used to determine the left and right states. Namely,

$$
\tilde{\mathbf{U}}_{L}=\tilde{\mathbf{U}}_{j}+\frac{1}{4}\left(I-\frac{\Delta t}{\Delta x} A_{j}\right)\left(\tilde{\mathbf{U}}_{j+1}^{n}-\tilde{\mathbf{U}}_{j-1}^{n}\right),
$$

and

$$
\tilde{\mathbf{U}}_{R}=\tilde{\mathbf{U}}_{j+1}-\frac{1}{4}\left(I+\frac{\Delta t}{\Delta x} A_{j+1}\right)\left(\tilde{\mathbf{U}}_{j+2}^{n}-\tilde{\mathbf{U}}_{j}^{n}\right) .
$$

Once $\tilde{\mathbf{F}}_{j+\frac{1}{2}}$ is calculated using Equation 3 , each of its terms is multiplied by the area $S$ at the flux interface location. The flux is now as in Equation $1 \mathrm{~b}$ and the conservative variables can updated using Equation 2. For the first order method, the source term used in Equation 2 is evaluated as

$$
\mathrm{T}_{j}=\mathrm{T}\left(\mathrm{U}_{j}^{n}\right),
$$

and for the second order method, the source term is determined using

$$
\mathbf{T}_{j}=\mathbf{T}\left(\mathbf{U}_{j}^{n+\frac{1}{2}}\right)
$$

where

$$
\begin{aligned}
\mathbf{U}_{j}^{n+\frac{1}{2}} & =\tilde{\mathbf{U}}_{j}^{n+\frac{1}{2}} S_{j} \\
& =\left[\tilde{\mathbf{U}}_{j}^{n}-\frac{\Delta t}{4 \Delta x} A_{j}\left(\tilde{\mathbf{U}}_{j+1}^{n}-\tilde{\mathbf{U}}_{j-1}^{n}\right)\right] S_{j} .
\end{aligned}
$$

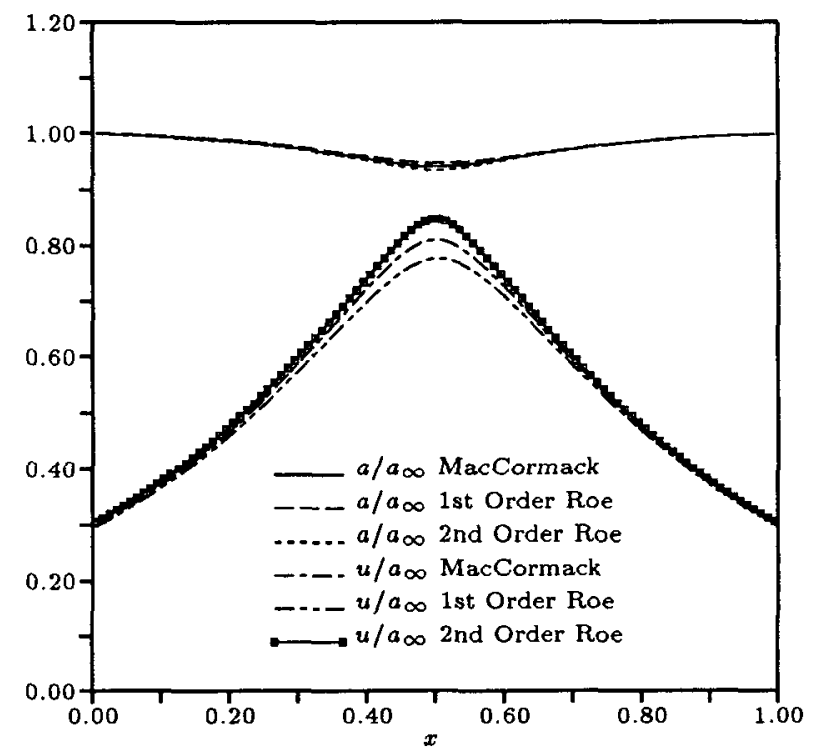

Figure 2: Steady State Solutions for Velocity and Sound Speed for the Various Nonlinear CFD Methods

\subsection{Nonlinear Results}

The steady flow solutions for each of the finite volume methods are seen in Figure 2, which shows computed velocity and sound speed distributions on a fixed mesh of 100 cells. Each individual method gives its own unique solution to the steady problem.

Figure $3 a$ shows results from MacCormack's method for the unsteady problem. For each frequency ${ }^{1}$, the envelope is plotted of the perturbation pressure as the wave passes down the channel. The code was allowed to run until each envelope reached an asymptotic limit. For the highest frequency, a sample pressure wave is also superimposed within the pressure envelope. As can be seen, up to $2000 \mathrm{~Hz}$, the acoustic wave is able to traverse the channel, however its strength is dissipated. For frequencies of $3000 \mathrm{~Hz}$ and higher, the acoustic wave can no longer make it past the midsection of the channel. Figure $3 \mathrm{~b}$ shows computed pressure distribution envelopes for the second-order upwind scheme. Again, the higher frequency signals are unable to traverse the channel. We therefore turn now to the development of novel schemes designed for minimal dissipation.

\section{The Upwind Leapfrog Scheme}

The leapfrog discretization for scalar advection $\left(u_{t}+a u_{x}=0 a>0\right)$ can be expressed as

$$
u_{j}^{n+1}=u_{j}^{n-1}-\nu\left(u_{j+1}^{n}-u_{j-1}^{n}\right),
$$

where the Courant number is $\nu=a \Delta t / \Delta x$. The stencil for this scheme is illustrated in Figure 4.

This three time level scheme is neutrally stable for all Courant numbers less than or equal to one and is there-

\footnotetext{
${ }^{1}$ The frequencies are defined in Hertz, assuming the nozzle to be one foot in length, with entry flow at standard sea-level conditions
} 


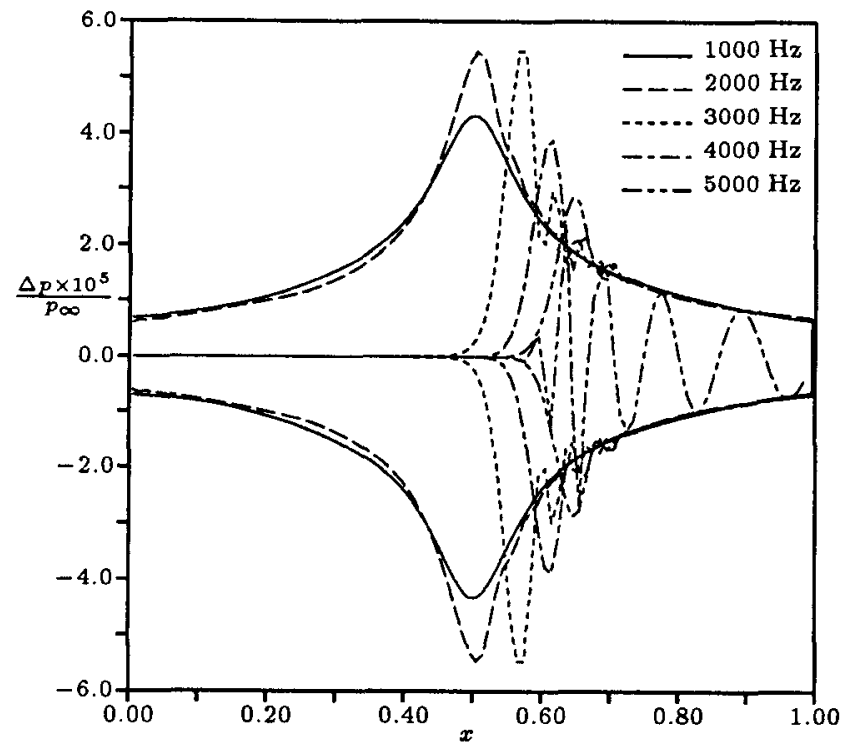

(a) MacCormack's Method

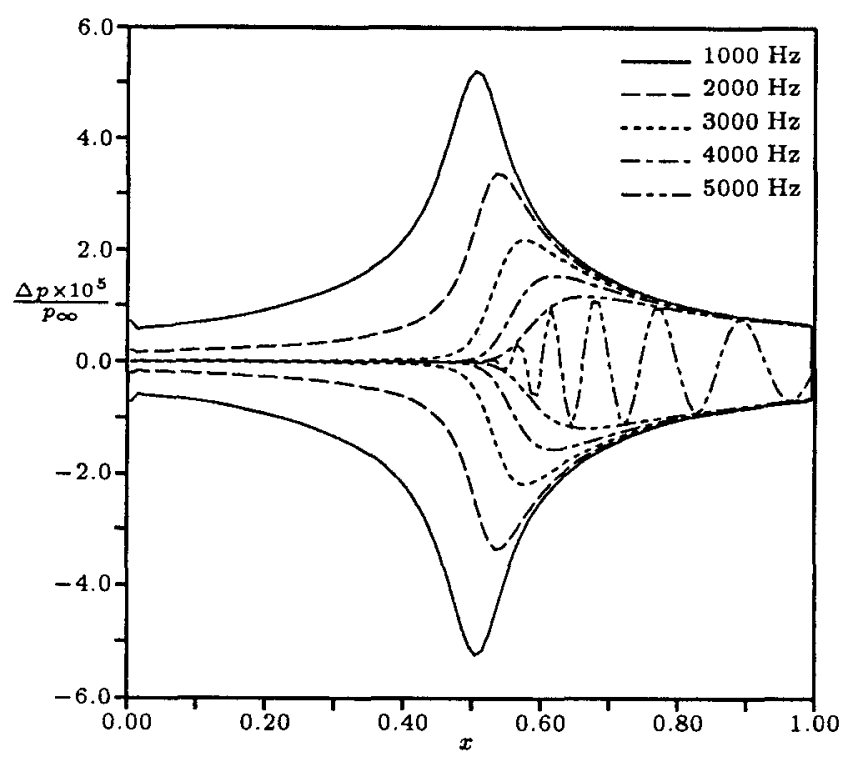

(b) Second Order Upwind Method

Figure 3: Pressure Wave Envelopes Using Dissipative Finite Volume Methods

fore free of dissipation error. However, it has very poor phase speed characteristics. This can be seen from Figure $5 \mathrm{a}$ where the difference between the numerical and exact wavespeeds, nondimensionalized by $\Delta x / \Delta t$,

$$
\nu_{\text {Numerical }}-\nu_{\text {Exact }}=\frac{\Delta t}{\Delta x}\left(a_{\text {Numerical }}-a_{\text {Exact }}\right),
$$

is plotted against $(N)$ the number of cells per wavelength. For all Courant numbers, as the number of cells per wavelength decreases, the difference between the numerical and exact wave speed increases. When the wavelength of the data is as short as is resolvable by the mesh $(2 \Delta x)$,

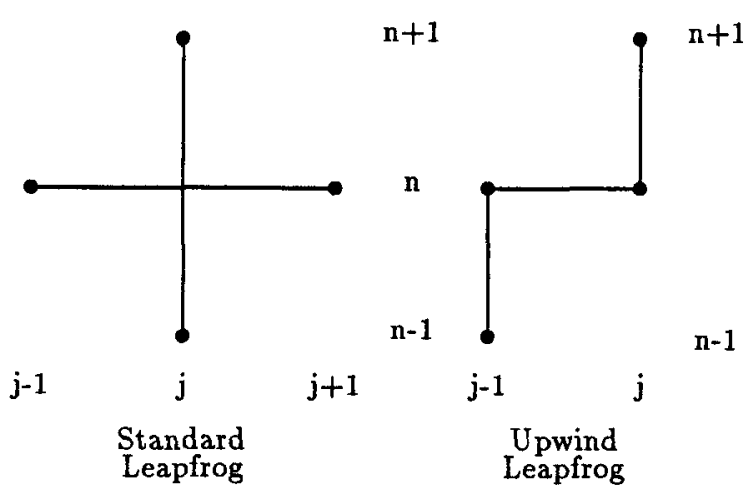

Figure 4: Computational Stencils for Standard and Upwind Leapfrog Schemes

the data will in fact no longer move. From Figure 5a, the number of cells required to maintain a certain level of accuracy may be easily deduced. For example, if one wishes to remain within $1 \%$ of the proper wave speed with a Courant number of 0.5 , at least 16 cells per wavelength will be needed.

Another three time level scheme for linear advection which also has neutral stability for all Courant numbers less than or equal to one is a scheme we have named the "upwind" leapfrog method. For an advection speed $a>0$, the discretization for linear advection is

$$
\frac{\left(u_{j}^{n+1}-u_{j}^{n}\right)+\left(u_{j-1}^{n}-u_{j-1}^{n-1}\right)}{2 \Delta t}+a \frac{u_{j}^{n}-u_{j-1}^{n}}{\Delta x}=0 .
$$

Here, the partial derivative of $u$ with respect to time $t$ is taken as the average of the backward time difference of $u$ at the mesh point $j-1$ and the forward difference at $j$. The stencil for this scheme is also illustrated in Figure 4, and the update at mesh point $j$ may be expressed as

$$
u_{j}^{n+1}=(1-2 \nu)\left(u_{j}^{n}-u_{j-1}^{n}\right)+u_{j-1}^{n-1} .
$$

When the advection speed $a<0$, a "mirror image" of the upwind leapfrog scheme (Equations 4 and 5) can be created. The corresponding update becomes

$$
u_{j}^{n+1}=(1+2 \nu)\left(u_{j+1}^{n}-u_{j}^{n}\right)+u_{j+1}^{n-1} .
$$

This scheme is the simplest member of a family of 'generalized leapfrog schemes' discussed by Iserles [8]. The reason this scheme is non-dissipative can be demonstrated with a simple argument. Suppose the scheme was not neutrally stable, but in fact dissipative with amplification factor $G$. If the integration proceeded backward in time, the amplitude factor would be $1 / G$. But in fact the forward and reversed time schemes are identical, as can be seen from the stencil that represents the scheme. If the the stencil is 'flipped' about the $\mathrm{n}$ time level, it is exactly the same stencil as it was before. Therefore $G=1$ and the scheme must be neutrally stable. A neutrally stable (and therefore at least second-order accurate) method can 


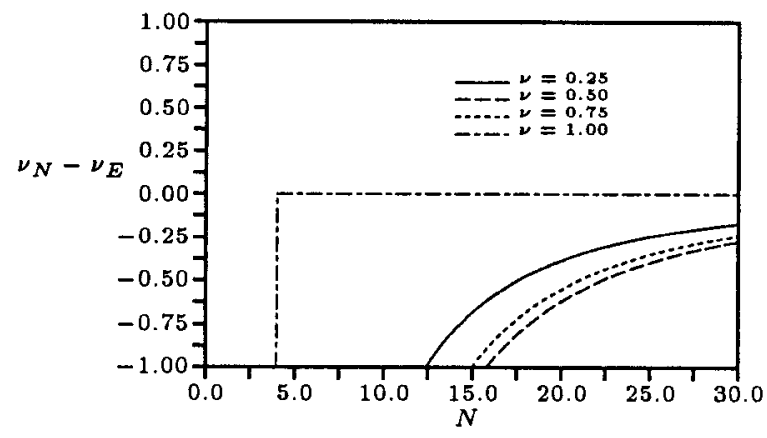

(a) Standard Leapfrog Method

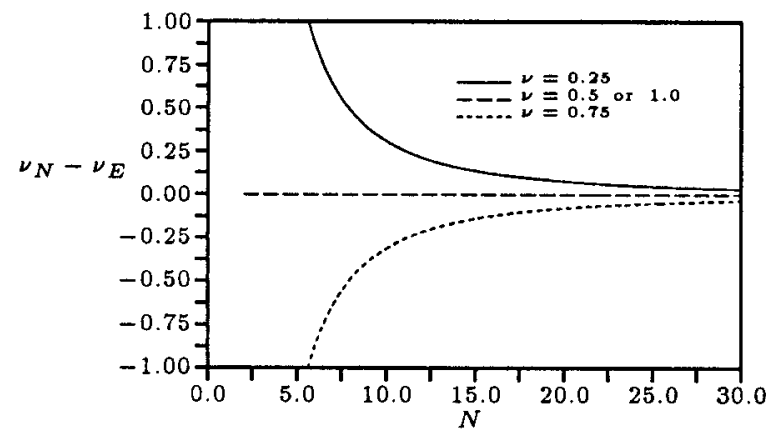

(b) Upwind Leapfrog Technique

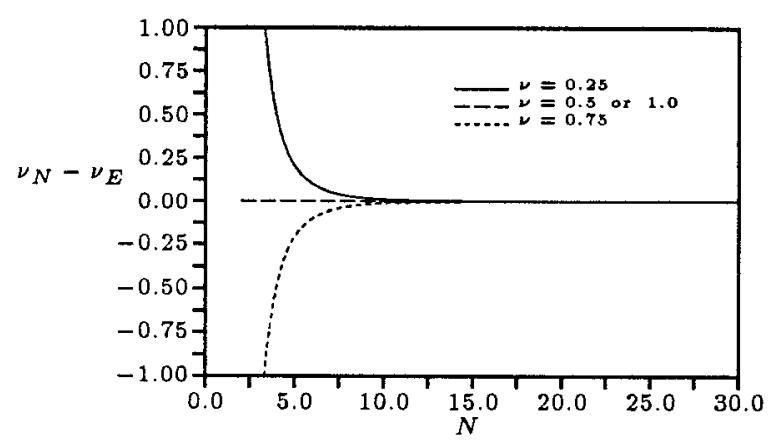

(c) Fourth Order Upwind Leapfrog Technique

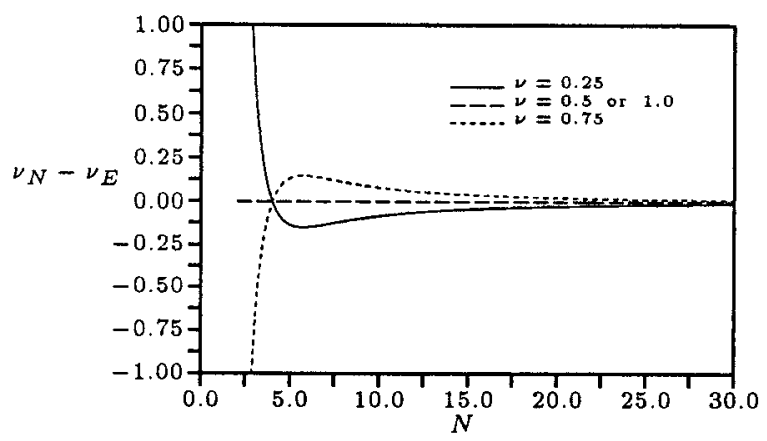

(d) Second Order Optimized Technique

Figure 5: Percent Error Between Numerical and Exact Wave Speeds for the Various Leapfrog Schemes

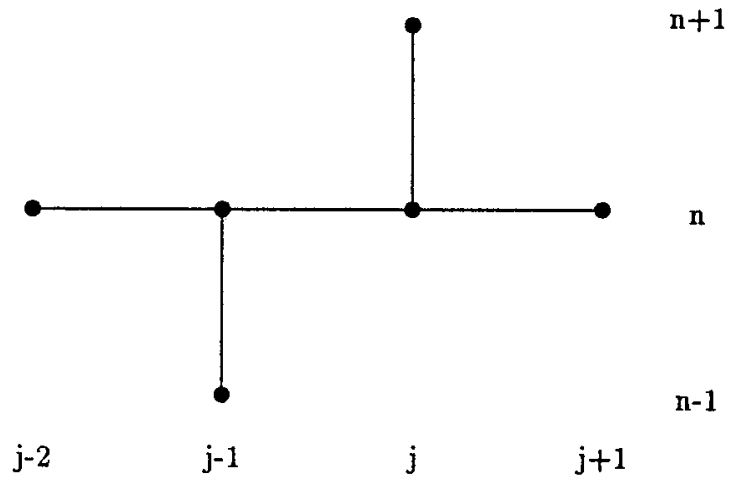

Figure 6: Stencil for the Fourth Order and Optimized Second Order Methods.

be created on any stencil that has a center of rotational symmetry.

Compared with the standard leapfrog technique, the upwind leapfrog scheme has much better phase speed characteristics, as can be seen from Figure 5b. The target of $1 \%$ phase error only requires $N \simeq 6$. Another potential advantage of this method derives from the compact stencil in space, which makes the application of characteristic boundary conditions extremely simple.

Another of Iserles' schemes uses a stencil shown in Figure 6. Again this stencil has rotational symmetry and the additional points $\left(u_{j-2}^{n}\right.$, and $\left.u_{j+1}^{n}\right)$ allow one to design a scheme which is fourth order accurate, or a scheme which has zero phase error for a specified number of cells per wave length. The spatial derivative in (4) is replaced by

$$
k \frac{u_{j+1}^{n}-u_{j-2}^{n}}{3 \Delta x}+(1-k) \frac{u_{j}^{n}-u_{j-1}^{n}}{\Delta x} .
$$

It can be shown that choosing

$$
k=\frac{1}{4}(1-2 \nu)(1-\nu)
$$

gives a scheme of fourth-order accuracy, and choosing

$$
k=\frac{3}{4 \nu}\left[\cos \frac{\nu \pi}{2}-\sin \frac{\nu \pi}{2}+2 \nu-1\right]
$$

gives a scheme that is free of error when $N=4$. Figures $(5 \mathrm{c}, \mathrm{d})$ show the remarkable accuracy of these methods. However, in our practical applications we have so far only implemented the second-order scheme. In the next section we will show how these upwind leapfrog schemes can be used to calculate acoutic perturbations of the nonlinear nozzle flow.

\section{$5 \quad$ Linearized Solutions}

\subsection{Primitive Variables}

There is no reason why the calculation of acoustic perturbations would have to be performed using the same 
code that computes the underlying steady flow. Furthermore, the acoustic calculation does not usually need to be conservative. Therefore we may use the primitive variable governing equations

$$
\frac{\partial \mathbf{W}}{\partial t}+C \frac{\partial \mathbf{W}}{\partial x}=\frac{\mathrm{D}}{S} \frac{d S}{d x}
$$

where

$$
\mathbf{W}=\left\{\begin{array}{l}
\rho \\
u \\
p
\end{array}\right\} C=\left[\begin{array}{ccc}
u & \rho & 0 \\
0 & u & \frac{1}{\rho} \\
0 & \gamma p & u
\end{array}\right] \mathbf{D}=\left[\begin{array}{c}
-\rho u \\
0 \\
-p u \gamma
\end{array}\right]
$$

\subsection{Linearization}

To linearize the primitive variable form of the governing equations, the acoustic small disturbance approximation is made for each of the primitive variables. Thus

$$
\begin{aligned}
\mathbf{W} & =\mathbf{W}_{0}(x)+\mathbf{W}_{1}(x, t) \\
& =\left\{\begin{array}{l}
\rho_{0} \\
u_{0} \\
p_{0}
\end{array}\right\}+\left\{\begin{array}{l}
\rho_{1} \\
u_{1} \\
p_{1}
\end{array}\right\}
\end{aligned}
$$

Substituting these relations into the primitive variable form of the governing equations, maintaining terms up to order $\rho_{1}, u_{1}$, and $p_{1}$, and observing that that the leading terms must cancel, the equations for the perturbation quantities become

$$
\frac{\partial \mathbf{W}_{1}}{\partial t}+C_{0} \frac{\partial \mathbf{W}_{1}}{\partial x}=\left(G \frac{1}{S} \frac{d S}{d x}-H\right) \mathbf{W}_{1}
$$

where

$$
C_{0}=\left[\begin{array}{ccc}
u_{0} & \rho_{0} & 0 \\
0 & u_{0} & \frac{1}{\rho_{0}} \\
0 & \gamma p_{0} & u_{0}
\end{array}\right]
$$

The source term due to area variation is

$$
G=\left[\begin{array}{ccc}
-u_{0} & -\rho_{0} & 0 \\
0 & 0 & 0 \\
0 & -p_{0} \gamma & -\gamma u_{0}
\end{array}\right]
$$

and the source term due to steady flow gradients is

$$
H=\left[\begin{array}{ccc}
\frac{d u_{0}}{d x} & \frac{d \rho_{0}}{d x} & 0 \\
-\frac{1}{\rho_{0}^{2}} \frac{d p_{0}}{d x} & \frac{d u_{0}}{d x} & 0 \\
0 & \frac{d p_{0}}{d x} & \gamma \frac{d u_{0}}{d x}
\end{array}\right]
$$

The matrices $C_{0}, G, H$ may be obtained from any available numerical solution to the nonlinear steady equations. In this work we use matrices derived from both the MacCormack and upwind solutions to the nozzle flow. In either case the problem to be solved is linear, although with non-constant coefficients. Although the presentation above is limited to one dimension, it is easily extended to more.

\subsection{Characteristic Form}

In order to devise a way to apply the upwind leapfrog scheme to the Euler equations, we begin by putting the linearized perturbation equations into characteristic form. If the boundary conditions do not introduce entropy perturbations then we assume that

$$
p_{1}=a_{0}^{2} \rho_{1}
$$

and eliminate $\rho_{1}$ from the equations. The two remaining equations can be put into characteristic form as

$$
\begin{aligned}
& \frac{\partial\left(p_{1}+\rho_{0} a_{0} u_{1}\right)}{\partial t}+\left(u_{0}+a_{0}\right) \frac{\partial\left(p_{1}+\rho_{0} a_{0} u_{1}\right)}{\partial x}=R^{+}(7) \\
& \frac{\partial\left(p_{1}-\rho_{0} a_{0} u_{1}\right)}{\partial t}+\left(u_{0}-a_{0}\right) \frac{\partial\left(p_{1}+\rho_{0} a_{0} u_{1}\right)}{\partial x}=R^{-(8)}
\end{aligned}
$$

where the right-hand sides are defined through

$$
\begin{aligned}
R^{ \pm}= & -\left[\left(a_{0}^{2} \rho_{0} \frac{1}{S} \frac{d S}{d x}+a_{0}^{2} \frac{d \rho_{0}}{d x}\right) \mp\left(\rho_{o} u_{0} \frac{d u_{0}}{d x}\right)\right] u_{1} \\
& -\left[\left(u_{0} \frac{1}{S} \frac{d S}{d x}+\frac{d u_{0}}{d x}\right) \pm\left(\frac{1}{\rho_{0} a_{0}} \frac{d p_{0}}{d x}\right)\right] p_{1}
\end{aligned}
$$

\subsection{Discretization}

The upwind leapfrog scheme is now applied to each of the characteristic equations as given by Equations 7 and 8 . The right-going characteristic equation is discretised (dropping the ()$_{1}$ subscripts) as

$$
\begin{aligned}
& \delta p_{j}^{n+\frac{1}{2}}+\rho_{0} a_{0} \delta u_{j}^{n+\frac{1}{2}}+\delta p_{j-1}^{n-\frac{1}{2}}+\rho_{0} a_{0} \delta u_{j-1}^{n+\frac{1}{2}}+ \\
& 2 \nu^{+} \Delta x\left(\Delta p_{j-\frac{1}{3}}^{n}+\rho_{0} a_{0} \Delta u_{j-\frac{1}{2}}^{n}\right)-2 \Delta t\left(R^{+}\right)_{j-\frac{1}{3}}^{n}=0
\end{aligned}
$$

and the left-going characteristic as

$$
\begin{gathered}
\delta p_{j}^{n+\frac{1}{2}}-\rho_{0} a_{0} \delta u_{j}^{n+\frac{1}{2}}+\delta p_{j+1}^{n-\frac{1}{2}}-\rho_{0} a_{0} \delta u_{j+1}^{n+\frac{1}{2}}+ \\
2 \nu^{-} \Delta x\left(\Delta p_{j+\frac{1}{2}}^{n}-\rho_{0} a_{0} \Delta u_{j+\frac{1}{2}}^{n}\right)-2 \Delta t\left(R^{+}\right)_{j+\frac{1}{2}}^{n}=0 .
\end{gathered}
$$

Here $\nu^{ \pm}$are defined by

$$
\nu^{ \pm}=\frac{\left(u_{o} \pm a_{o}\right) \Delta t}{\Delta x} .
$$

Solving these for the changes in the primitive variables at the currrent timestep gives

$$
\begin{aligned}
\delta p_{j, k}^{n+\frac{1}{2}}= & -\frac{1}{2}\left[\delta\left(p+\rho_{0} a_{0} u\right)_{j-1, k}^{n-\frac{1}{2}}+\delta\left(p-\rho_{0} a_{0} u\right)_{j+1, k}^{n-\frac{1}{2}}\right] \\
& -2 \nu^{+}\left[\rho_{0} a_{0} \Delta u-\Delta p\right]_{j-\frac{1}{2}, k} \\
& -2 \nu^{-}\left[\rho_{0} a_{0} \operatorname{div}+\Delta p\right]_{j+\frac{1}{2}, k} \\
\delta u_{j, k}^{n+\frac{1}{2}}= & -\frac{1}{2}\left[\delta\left(\frac{p}{\rho_{0} a_{0}}+u\right)_{j-1, k}^{n-\frac{1}{2}}-\delta\left(\frac{p}{\rho_{0} a_{0}}-u\right)_{j+1, k}^{n-\frac{1}{2}}\right] \\
& +2 \nu\left[\Delta u-\Delta p /\left(\rho_{0} a_{0}\right)\right]_{j-\frac{1}{2}, k} \\
& -2 \nu\left[\Delta u+\Delta p /\left(\rho_{0} a_{0}\right)\right]_{j+\frac{1}{2}, k}
\end{aligned}
$$

The amount of computation required is just slightly more than is involved in implementing a firstorder upwind scheme. 


\subsection{Upwind Leapfrog Nozzle Results}

For the test problem, both MacCormack's and Roe's methods have been used to generate the steady nonlinear solution. The upwind leapfrog method has then been applied to the resulting perturbation equations. Figure $7 \mathrm{a}$ shows the computed pressure wave envelopes based on MacCormack's steady solution results, and as can be seen, the acoustic wave is now able to completely move through the channel even at the higher frequencies, which are represented at the throat only by about 4 mesh points.

Figure $7 \mathrm{~b}$ shows the computed pressure wave envelopes based on the steady solution from the secondorder upwind method. Once again, by using the upwind leapfrog technique, the acoustic signals are able to traverse the length of the channel. Near the throat, the amplitudes are of course different from those predicted with the MacCormack solution as a basis, because the two nonlinear codes predict different Mach numbers there.

\section{A Multidimensional Advection Scheme}

For two-dimensional scalar advection $\left(u_{t}+a u_{x}+\right.$ $b u_{y}=0$ ) we can again create schemes free of dissipation by designing stencils with rotational symmetry. The simplest example is shown in Figure 8. This stencil is appropriate for wave directions bet ween zero and $90^{\circ}$. If we define

$$
\begin{aligned}
\Phi_{a b c d} & =\frac{a}{\Delta x}\left(u_{a}^{n}-u_{b}^{n}+u_{c}^{n}-u_{d}^{n}\right) \\
& +\frac{b}{\Delta y}\left(u_{a}^{n}-u_{c}^{n}+u_{b}^{n}-u_{d}^{n}\right)
\end{aligned}
$$

and

$$
\delta_{a}^{+}=u_{a}^{n+1}-u_{a}^{n} \text { and } \delta_{a}^{-}=u_{a}^{n}-u_{a}^{n-1}
$$

then for scalar advection, the corresponding discretization is

$$
\delta_{a}^{+}=\delta_{d}^{-}-2 \Delta t \Phi_{a b c d}
$$

Although this appears to be a natural way to extend the one-dimensional method, it has some drawbacks. One is that the algorithm changes discontinuously as the flow vector switches from one quadrant to the next. We have implemented versions that do not have this disadvantage, but only at the cost of reintroducing some small dissipation. The scheme from which we have so far had the best results does not actually exist in a scalar version, being based on bicharacteristic equations that inherently link more than one variable. This scheme will now be described.

\section{A Multidimensional Acoustic Scheme}

Consider the propagation of small amplitude waves in a uniform atmosphere. The flow can then be taken as

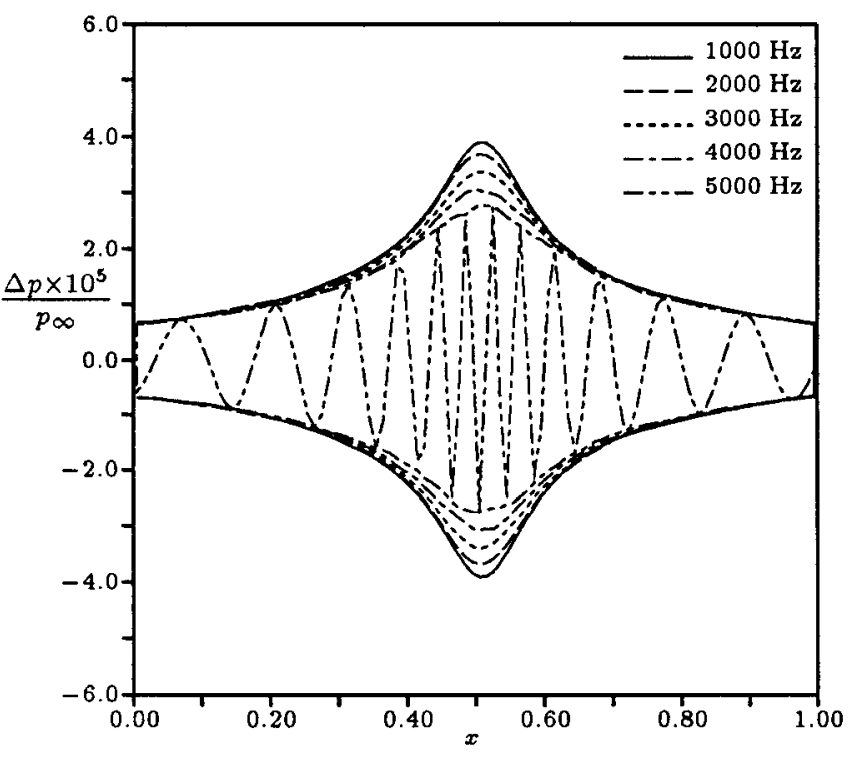

(a) Using MacCormack's Steady Flow Solution

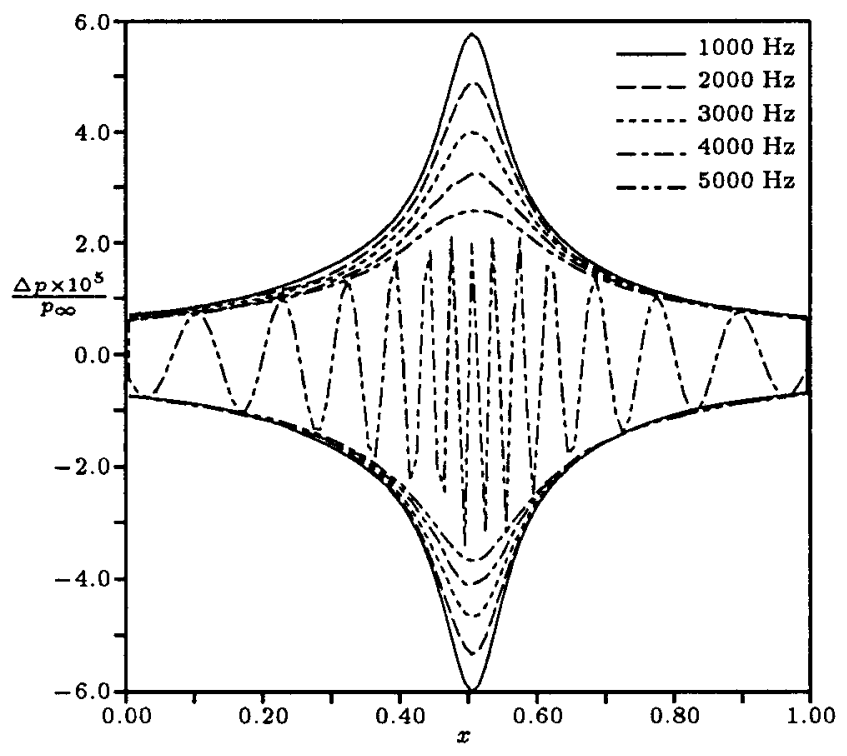

(b) Using Second Order Roe Steady Flow Data

Figure 7: Pressure Wave Envelopes Using Upwind Leapfrog Scheme

isentropic and the energy equation becomes redundant. For simplicity we take two dimensions, but add a source term to represent axial symmetry. The continuity and momentum equations are

$$
\begin{aligned}
\frac{\partial p}{\partial t}+\rho_{0} a_{0}^{2}\left(\frac{\partial u}{\partial x}+\frac{\partial v}{\partial y}\right) & =-\sigma \rho_{0} a_{0}^{2}\left(\frac{v}{y}\right) \\
\frac{\partial u}{\partial t}+\frac{1}{\rho_{0}} \frac{\partial p}{\partial x} & =0 \\
\frac{\partial v}{\partial t}+\frac{1}{\rho_{0}} \frac{\partial p}{\partial y} & =0
\end{aligned}
$$




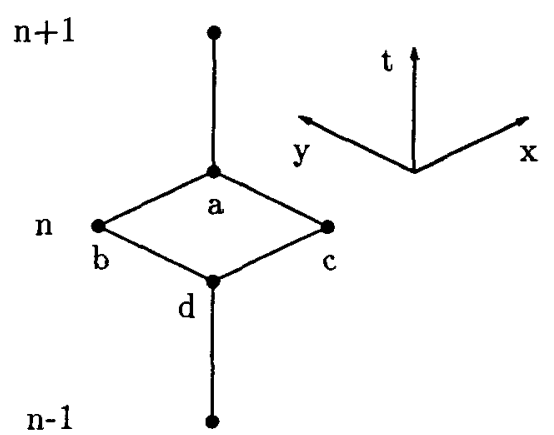

Figure 8: Stencil for Two-dimensional Upwind Leapfrog

where $\sigma$ is 0 for plane flow, and 1 for axisymmetric flow. Note that $v / y$ is a contribution to the velocity divergence; this fact will be used later.

A bicharacteristic equation is formed by adding these in the proportions $\left.\left(1, \rho_{0} a_{0} \cos \theta, \rho_{0} a_{0} \sin \theta\right)\right)$. This gives

$$
\begin{aligned}
& \left(\frac{\partial}{\partial t}+a_{0} \cos \theta \frac{\partial}{\partial x}+a_{0} \sin \theta \frac{\partial}{\partial y}\right)\left(p+\rho_{0} a_{0}(u \cos \theta+v \sin \theta)\right. \\
& =-\rho_{0} a_{0}^{2}\left[\left(\sin \theta \frac{\partial}{\partial x}-\cos \theta \frac{\partial}{\partial y}\right)(u \sin \theta-v \cos \theta)+\frac{\sigma v}{y}\right] .
\end{aligned}
$$

For any direction $\theta$, the $L H S$ resembles a one-dimensional characteristic equation, written along a generator of the Mach cone

$$
d x^{2}+d y^{2}=a_{0}^{2} d t^{2}
$$

and the RHS expresses derivatives tangential to that cone. The particular cases where the generator is contained in a coordinate plane are given by

$$
\begin{aligned}
& \frac{\partial\left(p+\rho_{0} a_{0} u\right)}{\partial t}+a_{0} \frac{\partial\left(p+\rho_{0} a_{0} u\right)}{\partial x}=-\rho_{0} a_{0}^{2}\left(\frac{\partial v}{\partial y}+\frac{\sigma v}{y}\right), \\
& \frac{\partial\left(p-\rho_{0} a_{0} u\right)}{\partial t}-a_{0} \frac{\partial\left(p-\rho_{0} a_{0} u\right)}{\partial x}=-\rho_{0} a_{0}^{2}\left(\frac{\partial v}{\partial y}+\frac{\sigma v}{y}\right), \\
& \frac{\partial\left(p+\rho_{0} a_{0} v\right)}{\partial t}+a_{0} \frac{\partial\left(p+\rho_{0} a_{0} v\right)}{\partial y}=-\rho_{0} a_{0}^{2}\left(\frac{\partial u}{\partial x}+\frac{\sigma v}{y}\right), \\
& \frac{\partial\left(p-\rho_{0} a_{0} v\right)}{\partial t}-a_{0} \frac{\partial\left(p-\rho_{0} a_{0} v\right)}{\partial y}=-\rho_{0} a_{0}^{2}\left(\frac{\partial u}{\partial x}+\frac{\sigma v}{y}\right) .
\end{aligned}
$$

This shows a typical difficulty in the application of bicharacteristic methods. Four equations emerge that are convenient to work with, but only three are needed to solve for the three unknowns. Good surveys of previous work on bicharacteristic methods can be found in $[9,10]$, and recent contributions in [11, 12].

Here, a straightforward generalization of our onedimensional method to discretize the first of Equations 12 would be to use the stencil shown at left in Figure 9. The time derivative is averaged over $F G, P Q$, and the space derivatives and source term are found using central differences over the shaded plane. The resulting equation has

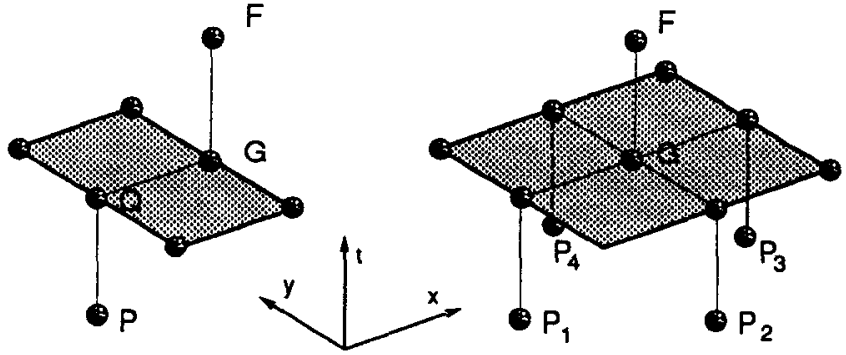

Figure 9: Illustrating a possible discretization of the bicharacteristic equations.

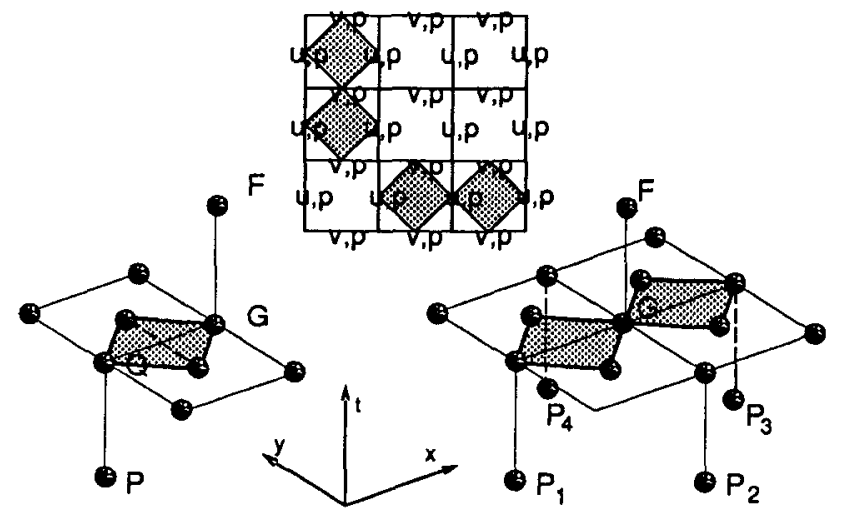

Figure 10: Illustrating the preferred discretization of the bicharacteristic equations.

symmetry around the midpoint of QG, and is therefore time-reversible.

The complete update at any mesh point can be found by assembling the four stencils oriented with $\theta=$ $0, \pm \pi / 2, \pi$. Inspection then shows that the bicharacteristic equations with $\theta=0, \pi$ determine the updates for $p, u$, and the bicharacteristics with $\theta= \pm \pi / 2$ determine the updates for $p, v$. This gives two independent updates for $p$ that may not agree. Averaging these destroys the time reversibility. In [11], consistency of the two updates was used to place a constraint on the spatial differencing, but that device is not available here.

Instead, we have resorted to a staggered mesh arrangement, in which the pressure is stored at twice as many locations as the velocities. There is a $50 \%$ storage overhead for doing this compared with using collocated variables, but the absence of dissipation should create savings that far outweigh this. At the top of Figure 10 the locations of variables on the grid can be seen. This is different from the conventional staggered arrangement in MAC codes [13] that places pressure at cell centers; to use a bicharacteristic method the variables that appear together on the LHS must be collocated. At bottom left is the stencil used to discretize the first of Equations 12, and at right the complete stencil used to update a point at which $(\mathrm{u}, \mathrm{p})$ are stored.

The final update scheme can be written quite sim- 
ply. On a uniform mesh it is, for a $(u, p)$ point,

$$
\begin{aligned}
\delta p_{j, k}^{n+\frac{1}{2}}= & -\frac{1}{2}\left[\delta\left(p+\rho_{0} a_{0} u\right)_{j-1, k}^{n-\frac{1}{2}}+\delta\left(p-\rho_{0} a_{0} u\right)_{j+1, k}^{n-\frac{1}{2}}\right] \\
& -2 \nu\left[\rho_{0} a_{0} \operatorname{div}-\Delta p\right]_{j-\frac{1}{2}, k} \\
& -2 \nu\left[\rho_{0} a_{0} \operatorname{div}+\Delta p\right]_{j+\frac{1}{2}, k}, \\
\delta u_{j, k}^{n+\frac{1}{2}}= & -\frac{1}{2}\left[\delta\left(\frac{p}{\rho_{0} a_{0}}+u\right)_{j-1, k}^{n-\frac{1}{2}}-\delta\left(\frac{p}{\rho_{0} a_{0}}-u\right)_{j+1, k}^{n-\frac{1}{2}}\right] \\
& +2 \nu\left[\operatorname{div}-\Delta p /\left(\rho_{0} a_{0}\right)\right]_{j-\frac{1}{2}, k} \\
& -2 \nu\left[\operatorname{div}+\Delta p /\left(\rho_{0} a_{0}\right)\right]_{j+\frac{1}{2}, k} .
\end{aligned}
$$

Here $\delta$ denotes a difference in time, and $\Delta$ a difference in $x$. We use 'div' to denote the undivided discrete divergence, (i.e. $h$ times the divergence) and this includes the $v / y$ term if axisymmetric flow is considered. Note that the first line in each equation is not computed, but merely recalled from the previous timestep. For a $(v, p)$ point, the formulae follow from a coordinate rotation. As with all multilevel methods, the first time step must be taken using a Lax-Wendroff or similar method.

At solid, possibly moving, boundaries, the normal velocity is prescribed, and the incoming bicharacteristic equation is solved for the pressure. This seems to be entirely satisfactory. At far-field boundaries, changes due to incoming bicharacteristics are set to zero. This causes some weak reflected waves, and is something that needs to be improved.

A von Neumann analysis of the scheme is possible, but does not lead to simple conclusions except in the special case $\nu=\frac{1}{2}$ (for which the one-dimensional scheme is exact). Discrete solutions of the form

$$
\mathbf{u}_{j, k}^{n}=\mathrm{U} \exp i\left(n \phi+j \theta_{x}+k \theta_{y}\right)
$$

exist, with the simple dispersion relationship

$$
\cos ^{2} \phi=\cos ^{2} \frac{\theta_{x}}{2} \cos ^{2} \frac{\theta_{y}}{2} .
$$

This includes the possibility of plane wave solutions crossing the grid at an angle $\alpha$, so that $\theta_{x}=\theta \cos \alpha$ and $\theta_{y}=\theta \sin \alpha$. For such a wave, we find

$$
\frac{\text { numerical wavespeed }}{\text { exact wavespeed }}=1-\left(\frac{\sin ^{2} 2 \alpha}{96}\right) \theta^{2}+\mathcal{O}\left(\theta^{4}\right) \text {. }
$$

With $N=6$, so that $\theta=\pi / 3$, plane wave propagation is isotropic to within about $1 \%$, for this particular CFL number. Experimentally we find that $\nu=\frac{1}{2}$ is the stability limit for this method when computing plane flow. Including the $v / y$ term in the divergence tends to create mild instability near the axis, and lowers the limit to about 0.48 . All the calculations reported here were performed with $\nu=0.40$.

\section{Piston Problem Results}

A good model problem for testing a numerical scheme's ability to handle multi-dimensional wave propa-
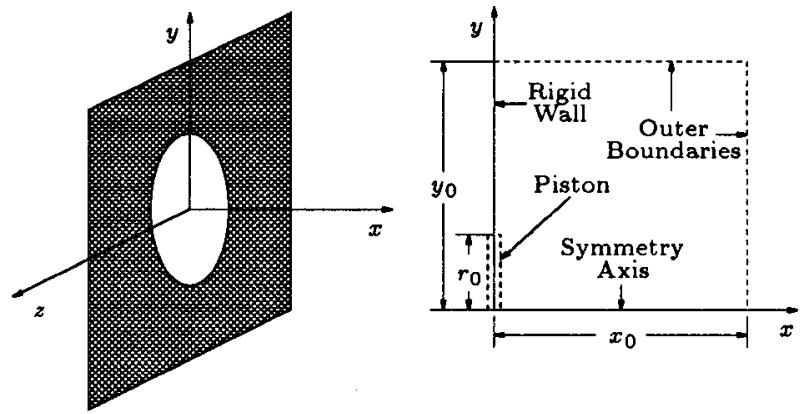

Figure 11: Geometry for Oscillation Piston Problem

gation is the simulation of the acoustic field produced by an oscillating piston over an infinite baffle (Figure 11).

It turns out that there exists an exact solution for pressure magnitude along the axis of symmetry. This is given by (Reference [14])

$$
\frac{p(x)}{2 \rho_{0} a_{0} U}=\left|\sin \left\{\frac{\pi f x}{a_{0}}\left[\sqrt{1+\left(\frac{r_{0}}{x}\right)^{2}}-1\right]\right\}\right| .
$$

Here, $U$ is the piston displacement magnitude, $f$ is its frequency, and $r_{0}$ is its radius. As can be seen, for a given $\rho_{0}, a_{0}$, and $U$, the axial pressure magnitude is only a function of $f, r_{0}$, and $x$. To illustrate the effectiveness of the upwind leapfrog technique, we fix $f$ and $r_{0}$, and vary the mesh sizes. Doing this allow us to change the number of mesh points per wave length and thus compare differences between various numerical techniques.

For our comparison simulations, we have chosen $f$ to be 10 cycles per unit time, and $r_{0}$ equal to $1 / 10$ of a unit length. The domain utilizes the symmetry of the problem and extends one unit in $x$ and $y$ directions. Mesh sizes of $200 \times 200,160 \times 160,120 \times 120$, and $80 \times 80$ correspond to simulations of approximately $20,16,12$, and 8 mesh point per wave length along the symmetry axis. The integrations are carried out until the wave reaches the far boundary. At later times our solution is contaminated by weak waves that reflect from the boundary. Such reflections are a problem for most numerical simulations and we plan to focus on this issue in future research.

When MacCormack's method is applied to this problem, (Reference [15]) it has been found that a minimum of 20 cells per wave length is required to get good results. Here we compare computations using Hancock's [7] finite volume technique with those from the upwind leapfrog method. Figure 12 shows computed axial pressure distribution magnitudes along with a sample wave from the coarsest mesh for the second order finite volume and upwind leapfrog techniques. Figure 12a shows how in addition to having difficulty following the correct pressure distribution magnitude near the piston face boundary, the finite volume technique damps out the wave on the coarser meshes as it recedes down the axis. However, by using the non-dissipative upwind leapfrog scheme, (Figure 12b), one can see that good results are 


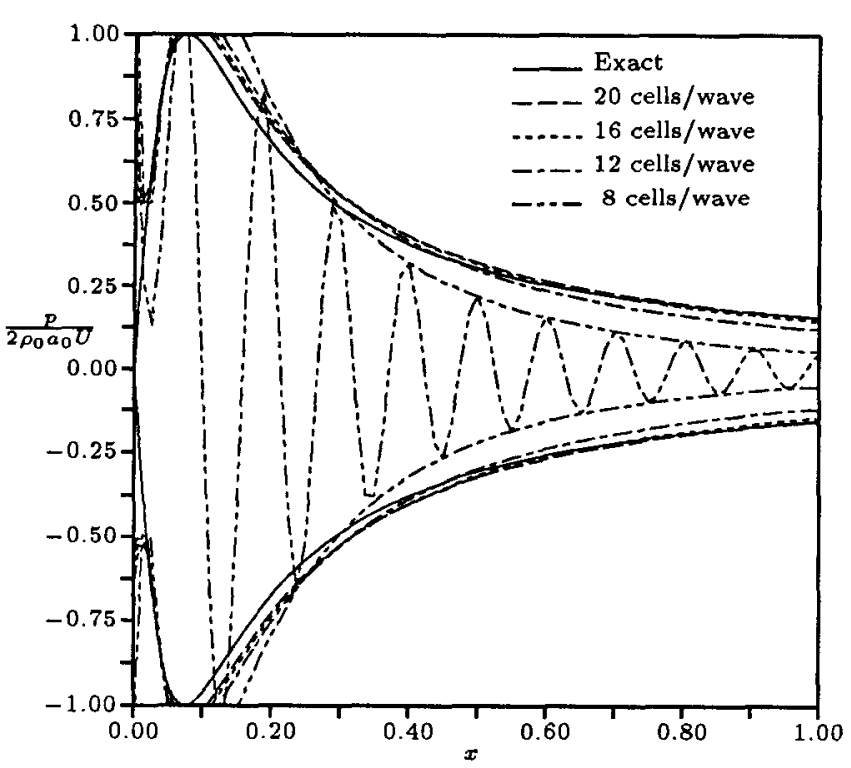

(a) Finite Volume Method

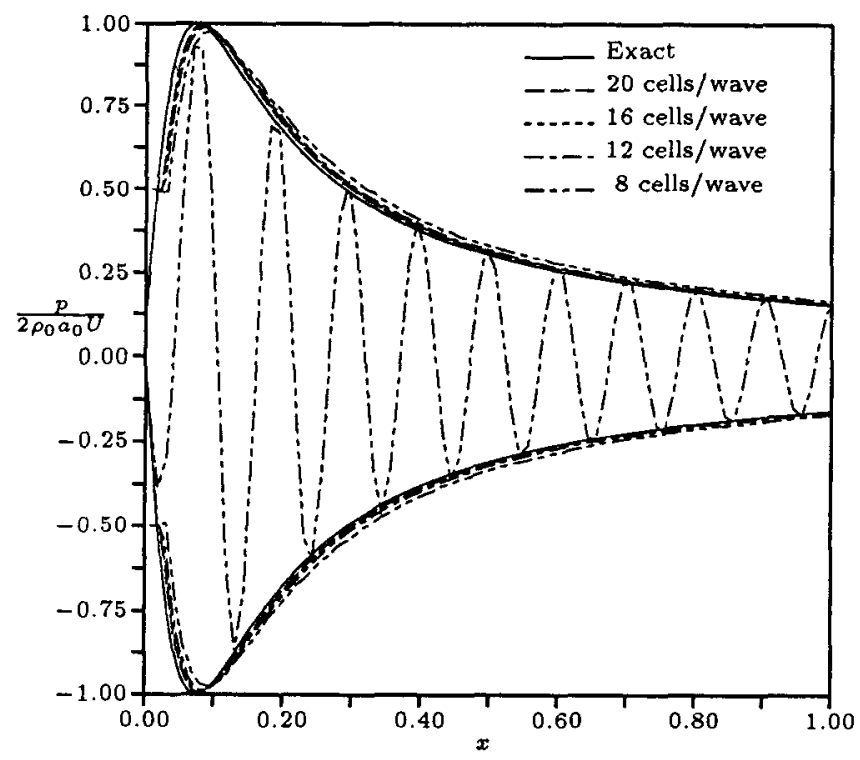

(b) Upwind Leapfrog Method

Figure 12: Computed Pressure Wave Envelopes for Various Mesh Sizes

possible with as few as eight cells per wave length.

In Figure 13 illustrates a case where a wave is propagated for 50 wavelengths (with $r_{0}=1 / 40$ unit length and $f=50$ ). The upwind leapfrog scheme, even over these large distances, yields a solution that follows the exact solution almost perfectly.

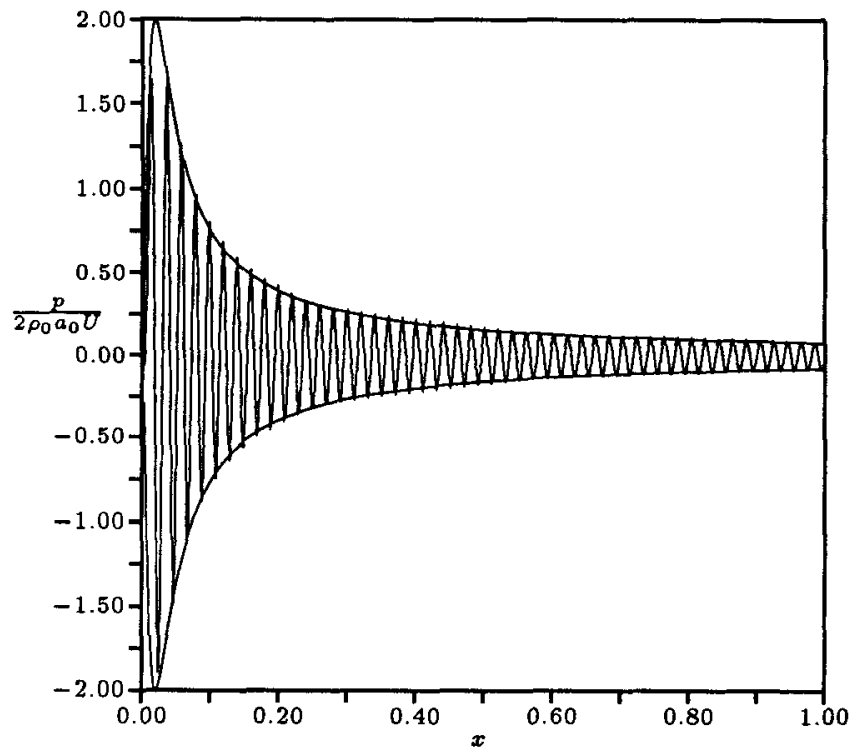

Figure 13: Pressure Wave Pattern and Envelope Using Upwind Leapfrog Technique - $400 \times 400$ mesh -8 Cells Per Wavelength

\section{Conclusions}

Standard aerospace CFD methods have been shown to be inadequate for even simple aeroacoustic problems unless excessively fine grids are used. The chief deficiency is a numerical dissipation that damps out the wave motions far from their source.

Dissipation can be avoided by employing schemes that are symmetrical in time, such as the classical leapfrog method. An upwind variant has been devised that has greatly reduced phase error. It has been shown how this can be used to simulate successfully the acoustic perturbation of a nonlinear nozzle flow. The mean flow may be derived from any existing CFD code.

An extension to two dimensional problems has been made that turns on a new handling of the bicharacteristic equations. A key point is that normal velocities are defined on cell edges and that pressure is defined twice as often as velocity. This avoids common problems such as having to average over the bicharacteristics, and allows the nondissipative properties to be preserved in higher dimensions. This method has been tested against a problem with an exact solution, and it seems that the waves can be propagated almost indefinately with losing energy.

We conclude that this work gives a very promising basis for the development of practical aeracoustic codes. In future research we will concentrate on the construction of nonreflecting boundary conditions, and on applying the method to multi-dimensional nonuniform mean flows.

\section{References}

[1] J. Lighthill. Report on the final panel discussion on computational aeroacoustics. ICASE Report 92-53, 1992. 
[2] D. A. Kopriva. Spectral solution of the time dependent Euler gasdynamics equations, AIAA paper DGLR/AIAA-92-02-036. In DGLR/AIAA 14th Aeroacoustics Conference, 1992.

[3] R. K. Agarwal, K. S. Huh, and M. Shu. A CFD based approach for the solution of acoustics, Maxwell, and Schroedinger equations for scattering problems. In 19th International Conference on Numerical Methods in Fluid Dynamics. Springer Verlag, 1993.

[4] R. W. MacCormack. The effect of viscosity in hypervelocity impact cratering. AIAA Paper 69-354, 1969.

[5] A. Jameson, W. Schmidt, and E. Turkel. Numerical solutions of the Euler equations by a finite-volume method using Runge-Kutta time-stepping schemes. AIAA Paper 81-1259, 1981.

[6] P. L. Roe. The use of the Riemann problem in finitedifference schemes. In 7th International Conference on Numerical Methods in Fluid Dynamics. Springer Verlag, 1980.

[7] G. D. van Albada, B. van Leer, and W. W. Roberts $\mathrm{Jr}$. A comparative study of computational methods in cosmic gas dynamics. Astronomy and Astrophysics, 108, 1982.

[8] A. Iserles. Generalised leapfrog methods. IMA Journal of Numerical Analysis, 6, 1986.

[9] P. I. Chuskin. Numerical methods of characteristics for three-dimensional supersonic flows. Progress in Aeronautical Sciences, 9, 1968.

[10] R. Camarero. Numerical Solution of Internal and External Flows at High Incidence. $\mathrm{PhD}$ thesis, McGill University, 1973.

[11] A. Dadone and G. Moretti. Fast Euler solver for transonic airfoils, Part I, theory. AIAA Journal, 26, 1988.

[12] I. H. Parpia, C. P. Kentzer, and M. H. Williams. Multidimensional time-dependent method of characteristics. Computers and Fluids, 16, 1988.

[13] F.H. Harlow and J. E. Welch. Numerical study of time-dependent viscous incompressible flow of fluid with free surface. Physics of Fluids, 9, 1965.

[14] L. E. Kinsler, A. B. Coppens, A. R. Frey, and J. V. Sanders. Fundamentals of Acoustics. John Wiley \& Sons, Inc., 1982.

[15] D. M. Nark. A computational aeroacoustics approach to sound generation by baffled pistons. Master's thesis, George Washington University, 1992. 